\title{
LE REPORTAGE IMMERSIF : UNE EXPÉRIENCE PARADOXALE DU RÉEL ET DE LA VÉRITÉ ?
}

\author{
Angelina Toursel ${ }^{1}$ et Philippe UseILlE ${ }^{2}$
}

\begin{abstract}
En quoi le reportage immersif propose-il une expérience paradoxale de la réalité et de la vérité dans un contexte marqué par une crise de confiance à l'encontre des médias ? L'immersion appliquée au journalisme propose au spectateur une expérience subjective de l'information qui associerait émotion, présence et transparence. Les journalistes qui y recourent espèrent que le public se sentira ainsi plus concerné, plus engagé. Mais, à sa manière, le journalisme immersif va au-delà et agirait comme un mode de véridiction qui interroge notre rapport à la réalité à l'ère dite de la « post-vérité ».
\end{abstract}

Dans un contexte marqué par la transformation rapide des médias et des pratiques de consommation de l'information, des innovations journalistiques expérimentent des modes de production et d'appropriation de l'information à travers de nouveaux « formats » médiatiques. Ils s'adressent à un destinataire invité à s'engager dans un travail multitâche de récepteur, d'interprète, d'interacteur ou de joueur (Aïm, 2013) qui façonnerait sa propre expérience informationnelle, à un moment où

1 Angelina Toursel est doctorante en sciences de l'information et de la communication au sein du laboratoire DeVisu de l'Université Polytechnique Hauts-de-France.

2 Philippe Useille est maître de conférences en sciences de l'information et de la communication au sein du laboratoire DeVisu de l'Université Polytechnique Hautsde-France.

Recherches en communication, $n^{\circ} 51$ - Article publié le 10/09/2020 
le journalisme est confronté à une crise de légitimité (Lancelin, 2018) et de confiance qu'enregistre chaque année en France le baromètre de Kantar/La Croix.

Le journalisme immersif, qui sera ici étudié à travers l'une de ses déclinaisons - le reportage immersif - se trouve au croisement de deux héritages : celui du reportage journalistique, soucieux de transporter le public in media res pour lui faire vivre l'actualité au plus près, et celui d'une tradition artistique qui établit un nouveau rapport à l'image. Or, cette rencontre n'est pas fortuite et parait même significative à l'heure où le journalisme, qui souffre d'un déficit de crédibilité, cherche à renouveler l'expérience de l'information.

Par journalisme immersif, nous ne parlons pas de cette forme d'investigation où le journaliste fait l'expérience personnelle d'une situation sur laquelle il enquête, telle que Günter Wallraff (1986) ou, plus près de nous, Florence Aubenas (2010) l'ont pratiquée, même si cette tradition ne doit pas non plus être ignorée dans le cas présent. L'expression « journalisme immersif» désigne ici les reportages qui recourent aux technologies immersives utilisées dans les salles de rédaction depuis seulement moins d'une décennie : réalité virtuelle en images de synthèse, réalité augmentée, réalité mixte et vidéo $360^{\circ}$. Pour visionner de tels reportages, on utilise couramment un smartphone, un ordinateur ou, de façon plus optimale, un casque de réalité virtuelle de type Oculus Rift ou PlayStation $V R^{3}$. Notre étude s'attachera aux reportages immersifs en vidéo $360^{\circ}$ qui renouvellent le reportage audiovisuel linéaire.

On pourrait se demander s'il n'est pas vain de s'attarder sur ce format médiatique qui risque de subir le sort d'autres formes éphémères de webjournalisme (Mercier \& Pignard-Cheynel, 2014). Pourtant, en changeant d'échelle temporelle, comme l'archéologie des médias nous y invite, le reportage immersif révèle quelques-uns des enjeux médiatiques et informationnels du journalisme actuel : comment configure-t-il un rapport paradoxal à l'actualité, au réel et à la vérité ?

Pour explorer cette question, nous sommes conduits à nous frayer un chemin à travers divers cadres conceptuels et théoriques permettant d'aborder plusieurs niveaux d'analyse complémentaires articulés aux études en journalisme. Premier apport, celui de la German Media Theory (Mersch, 2018) qui élabore une conceptualisation croisée du

3 La version commerciale de l'Oculus Rift, mis au point en 2012 par Palmer Luckey, a été lancée en 2016. Le casque PlayStation VR, compatible avec la console de jeux PlayStation 4, a lui aussi été commercialisé en 2016. 
médium, de la médialité (son principe) et de la médiation (son effectivité). Les processus de médiation appellent un médium qui est « cette matérialité qui se dérobe à la perception tout en constituant son véhicule » (Mersch, 2018, p. 257). La médiation " performe » la signification, alors même que ses effets masquent en retour l'acte même de cette performance. Mais le médium ne se limite pas à sa fonction de véhicule : il imprime sa marque, en altérant ou en transformant ce qui fait l'objet d'une telle médiation. Entre transparence et opacité, la médialité a donc une fonction épistémique, même si le plus souvent elle se fait imperceptible. Toutefois, le médium devient perceptible quand « sa matérialité se dérègle ou se détraque » (Mersch, 2018, p. 259). Or, le reportage immersif, comme forme médiale nouvelle et instable, instaure un dérèglement du sens et une dissonance que la philosophie des médias allemande peut nous aider à démêler. Deuxième apport : l'archéologie des médias. Selon Jussi Parikka (2017), cette approche, qui prend l'une de ses sources dans les travaux de Friedrich Kittler, vise à comprendre, à la manière de Michel Foucault, les conditions d'existence d'un dispositif de médialité dans une situation culturelle donnée. Or ces conditions d'existence sont non seulement discursives ou institutionnelles mais aussi liées à des configurations médiatico-techniques qui se laissent lire comme des archives pour Friedrich Kittler. Ainsi, l'archéologie des médias souligne le fait que les nouveaux médias « remédient» les anciens médias, que « les cultures médiatiques [sont] comme sédimentées en différentes couches » (Parikka, 2017, p. 33). L'archéologie du reportage immersif révèle les processus enfouis de sédimentation dont il est finalement une concrétion. Un tel dispositif de médiation postule un sujet dans une relation au réel et à la vérité, que la tradition philosophique, dernier apport et non le moindre, saisit dans leurs relations problématiques. En tentant de conjuguer de façon cohérente ces différentes filiations intellectuelles, cet article a pour objectif de penser le reportage immersif sous l'angle de l'expérience qu'il propose, du rapport au réel et à la vérité qu'il instaure, et d'apporter ainsi une contribution conceptuelle à une épistémologie du journalisme bousculée par l'apparition de tels objets hybrides qui en disent long sur les évolutions du journalisme contemporain. 


\section{Le reportage immersif ou comment concilier transparence des médias et distance de la médiation ?}

La singularité du reportage immersif réside dans la rencontre d'un projet journalistique et des possibilités offertes par une technologie. Comme nous y invite l'archéologie des médias, nous adoptons une « manière de réfléchir aux nouvelles cultures médiatiques en mettant à profit les intuitions tirées des nouveaux médias du passé, souvent en mettant l'accent sur les dispositifs, les pratiques et les inventions oubliées, bizarres, improbables ou surprenantes » (Parikka, 2017, p. 33). Le reportage immersif a aussi ses bizarreries et réserve des surprises qui sont autant de voies d'accès pour en comprendre les enjeux. Nous nous inspirons alors de la démarche suivie par Jonathan Crary, chercheur américain en histoire de l'art et en esthétique, dans son étude de la chambre noire sur une période historique qui va du $\mathrm{XVI}^{\mathrm{e}}$ au XVIII ${ }^{\mathrm{e}}$ siècle. Il l'examine dans son "statut mixte d'une image épistémologique prise dans un ordre discursif et d'un objet appartenant à une configuration de pratiques culturelles » (Crary, 2016, p. 65). La chambre noire relève selon lui d'un « système de représentation », d'une « structuration de l'observateur » et d'une « conception des rapports entre l'observateur et le visible » pris dans un " réseau d'énoncés et d'usages » (Crary, 2016, p. 67). Elle suppose l'isolement de l'observateur, son retrait du monde pour mieux le percevoir, une vision qui sépare l'acte de perception du corps physique de l'observateur supplanté par un dispositif mécanique (Crary, 2016, pp. 76-78).

Cette approche archéologique des médias appliquée au dispositif de la chambre noire pourrait s'étendre selon nous au reportage immersif en l'inscrivant dans toute l'épaisseur de l'histoire culturelle et des régimes de visualité antérieurs. On y décèlera de la même manière les processus d'attention et de perception à l'œuvre, les « techniques du corps » convoquées, les « manières d'entendre, de voir et de sentir en général » que suppose toute médialité (Parikka, 2017, p. 47). Le reportage immersif implique quant à lui et contrairement à la chambre noire, un modèle de subjectivité qui ne repose plus sur le clivage entre le sujet percevant et le monde perçu. Il dépasse même le primat de l'œil et de la vision dans la mesure où le corps lui-même est «médialisé » dans ses formes de perception et de sensation (Parikka, 2017, p. 65).

Comment cela se traduit-il ? Le reportage immersif répond à l'ambition ancienne de placer le spectateur non pas face à une image mais dans l'image, dans un dispositif illusionniste où le temps et l'es- 
pace ne font qu'un (Grau, 1999). On en trouvera diverses illustrations dans l'histoire : le panorama de Bourbaki peint à Lucerne en 1881, les fresques de Fra Andrea Pozzo du plafond de la cathédrale baroque Sant Ignazo à Rome (fin du XVII ${ }^{\mathrm{e}}$ siècle), ou, plus tôt encore, les fresques de la Casa dei Misteri à Pompei (60 av. J.-C.) célébrant un culte dionysiaque. Même si chacune de ces formes d'immersion poursuit des buts différents - religieux, militaires, ou initiatiques - il s'agit, dans tous les cas, d'accéder à un espace hors de portée. On retrouve même une certaine forme d'immersion dans le concept wagnérien de Gesamtkunstwerk (œuvre d'art total) ou dans Les Nymphéas de Claude Monet, installés selon les plans du peintre au musée de l'Orangerie. Monet disait qu'ils donnent « l'illusion d'un tout sans fin, d'une onde sans horizon et sans rivage ». L'histoire de l'immersion s'inscrit donc, en tant que promesse, dans une « série culturelle » (Gaudreault \& Marion, 2013) qui traverse l'histoire de l'art, mais aussi celle des innovations technologiques dont le reportage immersif est l'héritier.

Sur le plan technologique, ce type de vision s'est développé par exemple avec diverses « machines à voir » (Gleizes \& Reynaud, 2017), le stéréoscope, le cinérama, le stéréopticon, le photorama, et plus proche de nous, le cinéma IMAX qui cherche à produire une illusion mobilisant tous les sens. Au-delà de la seule vision, des expériences ont été faites dans les années 1960 par l'informaticien américain Ivan Sutherland dont le dispositif de simulateur de vol présente à l'utilisateur une image en perspective changeant à mesure qu'il se déplace. L'expérience de Sutherland conduit non plus seulement à être « dans » plutôt que « devant » l'image, mais aussi à circuler dans cette image, à interagir avec celle-ci, ce que favorisera plus tard le numérique. Le journalisme immersif a pris son essor au moment où ces technologies sont devenues accessibles pour le grand public. Le reportage audiovisuel, où le reporter " s'efface pour voir et entendre » (Brabant, 2012), se prêtait bien à une telle expérimentation.

Avec un recul de dix ans, de grandes tendances thématiques ${ }^{4}$ se dégagent concernant l'offre de contenu journalistique immersif. De nombreux reportages explorent des lieux clos ou difficilement acces-

4 http://www.digitalnewsreport.org/publications/2017/vr-news-new-reality/\#a-newsvr-proposition-to-win-tomorrows-audience 
sibles comme des prisons ${ }^{5}$, des camps de réfugiés ${ }^{6}$, des zones de crise ${ }^{7}$ ou de conflits armés ${ }^{8}$. On suit l'itinéraire de personnes en situation de vulnérabilité : des groupes entiers se déplaçant ${ }^{9}$ ou des portraits d'individus singuliers ${ }^{10}$. Le studio d'A1 Jazeera Contrast, lancé en 2017, s'est donné pour mission plus précise d'utiliser une « technologie immersive pour connecter les téléspectateurs aux communautés sous-représentées et mal représentées qui sont les plus touchées par les inégalités et les conflits $^{11} \gg$. Il s'agit de sujets dont on attend qu'ils suscitent de la part de l'utilisateur un engagement où l'émotion tient une place déterminante.

Les technologies immersives ont la réputation d'impliquer davantage et d'augmenter la charge émotive, ainsi que l'empathie ressentie par le destinataire qui les utilise (Ropert-Dupont, 2017). Les journalistes qui font le choix du reportage immersif espèrent que ce destinataire se sentira plus concerné, qu'il surmontera l'altérité qui le sépare de l'autre en partageant ses émotions, et en comprenant mieux sa situation dans la lignée d'autres formes, comme le cinéma ou le documentaire. Ils espèrent ainsi lui faire ressentir les conditions de perception de la personne filmée pour lui donner accès à son vécu. Le reportage immersif proposé au spectateur est présenté par ses promoteurs ${ }^{12}$ comme une expérience permettant de percevoir et de s'approprier l'actualité « à la première personne », de façon plus subjective, comme cela a été recherché en son temps par le newsgame (Useille, Lamy \& Blanchard, 2017).

5 6x9 : a virtual experience of solitary confinement - 360 video, The Guardian, 2016; https://www.theguardian.com/world/2016/apr/27/6x9-could-you-survive-solitaryconfinement-vr

6 Clouds Over Sidra, Nations Unis, 2014 ; http://unvr.sdgactioncampaign.org/cloudsoversidra/\#.XXyckmY69tQ

7 The Crossing: A $360^{\circ}$ Look Into the Journey of Refugees to Greece, Huffington Post/ Ryot, 2015.

8 Yemen's Skies of Terror, d'Al Jazeera Contrast, 2018 ; https://contrastvr.com/yemen

9 Refugees 360 VR documentary - Scopic, 2015 ; http://scopic.nl/news/2016/4/12/ awarded-best-european-independent-experimental-film

10 The Displaced | 360 VR Video | The New York Times, 2015 ; https://vrfilmreview. $\mathrm{ru} /$ nytimes-vr-the-displaced-d9a1bd40de58

$11 \mathrm{https}: / /$ medium.com/ajcontrast/how-diversity-impacts-storytelling-an-all-femalenews-team-tells-all-47177c2c1383 : "use immersive technology to connect viewers to the underrepresented, misrepresented communities hit hardest by inequality and conflict".

12 https://www.cjr.org/tow_center/virtual-reality-study-engagement.php 
Rapprocher le destinataire de la personne filmée, lui faire comprendre sa situation et ses motivations et toucher in fine le destinataire pour susciter son désir de s'informer, voire son engagement : telles sont les vertus prêtées au reportage immersif, comme l'explique Chris Milk, réalisateur de Clouds Over Sidra (2015) consacré à la crise des réfugiés syriens, lors d'une conférence TED intitulée «Comment la réalité virtuelle peut créer l'ultime machine à empathie ${ }^{13}$ ? »

Selon la journaliste américaine Nonny de la Peña, l'une de ses pionnières, le journalisme immersif favoriserait un phénomène de « présence » dans la situation, le fait de se sentir avec tout son corps dans « l'histoire » (De la Peña et al., 2010). Plusieurs études empiriques ont été menées pour mesurer cet effet de présence et ses conséquences, comme en rend compte, par exemple, le rapport rédigé par The media impact project of the USC Annenberg Norman Lear Center (Karlin et al., 2018). Le rapport souligne que les participants, bien qu'immergés, ressentent l'incapacité - frustrante pourrait-on dire - d'interagir avec leur environnement.

Nous avons voulu en savoir plus. En quoi consiste cette présence invoquée par Nonny de la Peña ? C'est en effet à travers cette notion problématique que se noue en partie le rapport paradoxal à l'actualité, au réel et à la vérité configuré par le reportage immersif. Notre étude de cas, consacrée au reportage en $360^{\circ}$ The Fight for Falluja de Ben C. Solomon du New York Times ${ }^{14}$ selon une méthode croisant phénoménologie et sémio-pragmatique, a permis de parvenir à certaines conclusions provisoires (Toursel \& Useille, 2019). Du côté du destinataire, l'effet de présence ressenti - le fait de se trouver au milieu des combattants sur le toit d'une maison de Falluja et de pouvoir explorer du regard cette scène - va de pair avec le sentiment d'être attaché à un point fixe, celui occupé par la caméra $360^{\circ}$ qui définit les coordonnées de « 1'ocularisation », c'est-à-dire du point de vue, au sens strictement visuel (Jost, 1987). Le destinataire s'éprouve comme pur regard désincarné, car dépourvu de corps, suspendu dans les airs en quelque sorte. De plus, même s'il se tient à côté des autres personnages, il ne peut communiquer avec eux.

13 How virtual reality can create the ultimate empathy machine? Conférence TED (2015) : https://www.youtube.com/watch?v=iXHil1TPxvA\&t=485s

14 https://www.nytimes.com/interactive/2016/08/14/magazine/fight-for-falluja-vr. html 
Quant au journaliste, il semble en retrait. Le reportage immersif propose une vision sphérique radicalement autre par la dissolution du cadre comme espace de représentation, ce qui n'est pas sans conséquence sur l'éthos du journaliste, lequel se manifeste, entre autres, par le choix du point de vue et du cadrage. Dans le reportage immersif, le réel semble être perçu sans médiation, en l'absence - à première vue - d'un principe organisateur (Gaudreault \& Jost, 2005, p. 25) qui guiderait le regard. L'opérateur, qui en a pourtant d'abord choisi la localisation, s'efface au profit de la caméra $360^{\circ}$ qui donne l'illusion d'une " monstration » neutre, condition de la transparence du reportage. L'effacement du journaliste serait-il la condition de la présence du destinataire ? Cette ambition n'est pas nouvelle et a déjà été exprimée lors du développement de l'information télévisée par exemple. Thierry Lancien (2011) montre qu'elle a été dominée au début des années 1960 par le culte de l'événement, du direct et du reportage. Avec le reportage immersif, l'apparent effacement de la figure du journaliste, renforcée par une médialité visant la transparence, alimente une « illusion scientiste du fait brut » mise en évidence par Daniel Cornu (1998, p. 16). Cet auteur distingue trois fonctions dévolues au journaliste : observateur, interprète et narrateur de l'actualité. Dans le dispositif immersif, le Sujet-spectateur est directement convié à exercer partiellement les fonctions d'observateur et davantage encore d'interprète, car le journaliste lui donne à voir et à interpréter ce qu'il a observé. Quant à la troisième fonction du journaliste, la fonction de narrateur, elle s'exerce selon Daniel Cornu dans la « véridicité du récit » et l'« authenticité du narrateur » (Cornu, 1998, p. 23). Mais, note-t-il aussi :

Les journalistes se donnent pour tâche de reconstruire par l'observation le monde de ce qui est. Ils tentent parfois de dire par l'interprétation le monde de ce qui doit être. Ils sont souvent déficients lorsqu'il s'agit de représenter par le témoignage le monde de ce qui est éprouvé (Cornu, 1998, p. 24).

Face à cette déficience ou difficulté, le journaliste trouve dans la fonction narrative " la capacité de dire ce qui est éprouvé » (Cornu, 1998, p. 24). Les technologies immersives apportent une solution nouvelle. La fonction de témoin serait étendue au spectateur qui verrait l'événement tel que le journaliste le voit et peut potentiellement le voir en tournant son regard dans toutes les directions : l'authenticité 
du témoignage pourrait être validée par le spectateur, témoin du même événement car plongé dans le fait. Il devient alors lui-même en partie narrateur de sa propre expérience par le choix de tourner son regard où il veut, même si le fait présenté reste limité à ce que le journaliste a filmé grâce à la caméra $360^{\circ}$. Avec les reportages immersifs, on assiste à une extension au Sujet-spectateur des trois fonctions du journaliste établies par Cornu, à savoir, l'observation, la compréhension et la narration de la réalité.

Cependant, cette impression d'effacement et de transparence du point de vue journalistique est brouillée et « se détraque » dans les séquences suivantes du reportage The Fight for Falluja. Divers indices révèlent les choix et les décisions du journaliste : la localisation et le déplacement de la caméra, le montage, l'incorporation d'informations textuelles, la musique, la voix off du commentaire, autant de manifestations d'une instance structurant le matériau filmique. Dans le reportage immersif étudié, qui reste une forme instable et hétérogène, la médiation fait donc alterner transparence et distance, provoquant une sorte de dissonance médiatique. D'une part, le destinataire semble faire l'expérience du monde « réel » sans médiation, comme l'a vécu le reporter lui-même (Le Cam \& Ruellan, 2017), et peut-être même dans la vérité de l'émotion qu'il a ressentie. Par sa présence dans l'image à l'emplacement même de la caméra, le destinataire vivrait à son tour les émotions du reporter, dont les indices cependant n'apparaissent pas dans les plans : il n'y a pas d'effet de bougé par exemple ; la caméra, à laquelle une place est attribuée, ne peut traduire les mouvements du cameraman. D'autre part, la distance de la médiation est assumée par l'instance narrative qui configure l'espace-temps du reportage et le commente en voix off. Ainsi, ces deux dimensions interagissent : la distance est intégrée dans la transparence. La présence du destinataire se justifie comme le vecteur organisateur de ces deux dimensions, voire comme instance d'authentification de ce qui a été rapporté par le journaliste.

Avant d'en faire la monstration ou la description, le reportage « déclare un événement », dit que quelque chose a eu lieu. Il « fait acte » et nous incite à « prendre acte » (Beyaert-Geslin, 2009, p. 41). Comme il pousse le destinataire à interagir avec un environnement, et à se mouvoir dans l'image, le reportage immersif efface ce qui séparait le « eux-ailleurs-alors » du « je-ici-maintenant ». Il nous transporte dans un espace autre (fait souvent souligné dans le cas des environnements immersifs). Mais il rapproche aussi le passé (vécu et capté par le reporter) et le présent du destinataire : « il s'agit bien de fonder un champ de 
présence commun associant énonciateur et observateur dans la même expérience du monde contemporain » (Beyaert-Geslin, 2009, p. 46). Le reportage immersif propose en effet au destinataire une actualité « à vivre $»$.

Une telle expérience « à vivre » produit un « champ de présence commun » qui authentifie les traces auxquelles est confronté le journaliste : des témoignages de première ou de seconde main, des communiqués, des déclarations, des « discours sur» (Cornu, 1998, p. 15). Si ces traces sont récentes et discutables, elles engagent la responsabilité du journaliste et le confrontent à « la modestie de la vérité de fait et l'inévitable conflit des interprétations » (Cornu, 1998, p. 15). L'expérience subjective que fait le destinataire d'une actualité grâce à l'immersion permet cette authentification des traces recueillies par le journaliste qui font l'objet de traitements dans le respect des codes et des normes du journalisme. Le reportage immersif met en scène la vérité des traces en convoquant l'expérience du destinataire qui les authentifie en les éprouvant. L'expérience immersive performe en quelque sorte l'authenticité du discours journalistique - toujours fragile - en conciliant transparence des médias et distance de la médiation selon un paradoxe médiatique inédit dans la mesure où le corps est engagé dans la représentation et en est même le médium. En cela, l'immersion, telle qu'elle est mise en œuvre dans le reportage immersif, a une valeur épistémologique dans la manière dont elle repose la relation triangulaire Sujet-réel-vérité.

\section{Le reportage immersif : un journalisme de la « post-vérité »?}

La German Media Theory met en évidence la médialité d'un objet par une attention portée au " comment des pratiques, leurs modalités spécifiques et leur performativité, ainsi que les effets qu'ils déclenchent » (Mersch, 2018, p. 13). Comprise ainsi, la médialité du reportage immersif se présente comme un moyen d'accès à une réalité hors de portée : elle est supposée agir sur le destinataire de telle sorte qu'il ait l'impression de vivre un fait d'actualité qui s'est produit « ailleurs et alors » et qu'il puisse certifier son authenticité.

Pourtant, cette impression est fragile car elle conjugue une double expérience avec des degrés variables d'implication : celle bien réelle du destinataire, engageant son esprit et son corps, et celle des personnes impliquées dans l'événement. Les discours des auteurs de reportages immersifs cultivent une certaine ambiguïté quand ils évoquent l'expé- 
rience permise par le dispositif immersif, car ils confondent plusieurs niveaux de réalité et d'expérience : s'agit-il de l'expérience réelle, « ici et maintenant », de l'utilisateur au moment où il visionne le reportage ? S'agit-il plutôt de l'évènement réel qui a eu lieu « ailleurs et alors »? Ou en dernier lieu, de ce que produit le journaliste-témoin à partir de son expérience de la situation, à savoir : une représentation en mode immersif de cet événement ? L'interaction, voire la confusion entre le réel et sa représentation, thème somme toute assez classique et déjà discuté en ce qui concerne les œuvres artistiques ou fictionnelles, pose de nouveaux problèmes en journalisme où la question de la vérité, étroitement liée à celle du réel, s'en trouve bousculée. Nous considérons le journalisme comme un mode de production de savoirs sur le réel : c'est même «l'une des institutions les plus influentes en matière de production de savoirs dans notre société ») (Ekström \& Westlund, 2019a, p. 1) ${ }^{15}$. Interroger le mode de vérité revendiqué explicitement ou implicitement par ces reportages immersifs relève d'un questionnement épistémologique portant sur la nature, le sens et la valeur accordés à l'information journalistique.

On s'accorde généralement à faire de la vérité le principe premier guidant la pratique des journalistes (Kovach \& Rosenstiel, 2014). La vérité est aussi une visée épistémologique « obligée » de l'information journalistique (Gauthier, 2004). De l'allégation de vérité dépend l'autorité du journalisme (Carlson, 2016). La vérité est en plus une exigence du consommateur d'information : « les allégations de vérité [...] sont évaluées, acceptées ou rejetées dans les pratiques de consommation de ces actualités » (Ekström \& Westlund, 2019b, p. 260) ${ }^{16}$. Fin ultime du journalisme, la vérité à laquelle il se réfère est pourtant modeste selon Daniel Cornu, puisque « l'information journalistique s'occupe de la vérité de fait »(Cornu, 1998, p. 14). En journalisme, la vérité de fait renvoie à une théorie de la vérité-correspondance comprise comme l'adéquation du discours à la réalité (Marian, 2016). Mais le reportage immersif apparaît dans le contexte dit de «post-vérité », caractérisé par la moindre importance accordée à une vérité d'ordre rationnel et par la préséance de l'émotion sur celle-ci (Ferraris, 2018). La philosophe Myriam Revault d'Allonnes insiste sur les conséquences politiques et

15 Notre traduction de « Journalism is among the most influential knowledge-producing institutions in modern society » (Ekstrom \& Westlund, 2019a, p. 1).

16 Notre traduction de « truth claims are [...] evaluated, accepted or rejected in the practices of news consumption » (Ekström \& Westlund, 2019b, p. 260). 
sociales de la « faiblesse du vrai » (Revault d'Allonnes, 2018) : l'abolition de la valeur normative du vrai signe la fin de la référence à une vérité de fait de sens commun sur laquelle sont arrimées les opinions dans les débats démocratiques; les responsables politiques sollicitent davantage les émotions et les croyances que la rationalité des citoyens lorsqu'ils s'adressent à eux. À partir de l'étude généalogique de ce phénomène de " post-vérité ", le philosophe Alain Cambier considère que celui-ci est un renoncement, qu'il « apparait comme une involution, un retour en arrière concernant les critères pour distinguer le vrai du faux » (Cambier, 2019, p. 8), alors même qu'une crise de confiance touche les « sachants », parmi lesquels les journalistes, supposés porter une parole de vérité (Mercier, 2018). En dépit de cette suspicion, l'attachement à la véracité demeure, ces deux attitudes étant liées comme le souligne le philosophe Bernard Williams : «le désir de véracité induit un processus critique qui fragilise l'assurance qu'il y aurait une vérité sûre ou qui se puisse affirmer sans réserve » (Williams, 2006, p. 13).

$\mathrm{La}$ « post-vérité » renvoie à une nouvelle conception de la vérité : certes, comme valeur, elle fait encore sens, tout comme le rapport d'adéquation caractérisant la vérité-correspondance, mais les critères communément admis pour la délimiter se seraient déplacés. Pour être dans le vrai, il ne suffirait plus de produire et de transiter par des discours rationnels en adéquation avec le réel, il faudrait plutôt privilégier le mode de l'expérience et de l'émotion vécue subjectivement pour saisir une réalité.

Symptômes ou conséquences, les manifestations de cette tendance de fond se retrouvent dans les reportages immersifs. Ces productions ne constituent-elles pas une sorte de réponse à la crise du journalisme et à la défiance du public caractérisant cette ère dite de la " post-vérité »? Toujours est-il que les discours qui accompagnent les reportages immersifs prétendent qu'ils font vivre le fait d'actualité dans toute la vérité de l'émotion qu'il suscite, rejoignant ainsi l'un des aspects prégnants de cette ère de " post-vérité ». Pour intensifier l'empathie spectatorielle, le reportage immersif s'appuie sur une mise en scène d'ordre affectif: " le déclenchement de sensations, d'émotions, a pour but d'accroître le réalisme ou l'effet de réel des images, et par là même leur degré de véracité » (Ropert-Dupont, 2017, p. 111). Par exemple, on trouve sur la plateforme Within, une production tournée en caméra $360^{\circ}$, représentative des thèmes fréquemment explorés: This is Climate change: 
famine $^{17}$. Ce reportage n'aborde pas cette crise alimentaire à travers les statistiques impersonnelles maintes fois entendues annonçant une famine de plus quelque part dans le monde, et où les êtres humains qui en sont frappés s'évanouissent dans l'objectivité des nombres. Il propose plutôt, grâce à l'immersion, de rencontrer les victimes, d'éprouver leur souffrance, de se tenir auprès d'individus singuliers que le reportage immersif arrache à l'anonymat statistique (Boltanski, 2007).

Cette approche n'est pas nouvelle : cette « pratique intensive du terrain » est commune aux sciences sociales et au journalisme (Leroux $\&$ Neveu, 2017). L'immersion en journalisme compte des figures majeures : James Greenwood (1866) qui s'est métamorphosé en vagabond pour pénétrer dans un refuge pour démunis, Nellie Bly (1887) qui a réussi à se faire interner incognito dans un asile psychiatrique pour les besoins de son enquête (Leroux \& Neveu, 2017, p. 14), Stephen Crane qui pratiquait l'experiment consistant « pour le journaliste, à se glisser temporairement dans la peau d'un autre pour se mettre dans la position d'expérimenter des réalités étrangères au quotidien de la plupart des lecteurs » (Vanoost, 2017, p. 39). La pratique de l'immersion dans le journalisme est « une forme physiquement et souvent politiquement engagée du reportage » qui recourt à l'expérience vécue dans un monde méconnu, devenant un " outil privilégié de rapport au réel, à sa vérité » (Leroux \& Neveu, 2017, p. 13). Le dévoilement de ce qui semble hors d'atteinte autrement s'accompagne alors d'un changement de regard sur cette réalité.

Cependant, dans le cas du reportage en vidéo $360^{\circ}$, l'immersion concerne le spectateur lui-même. Comment la médialité de ce reportage immersif agit-elle? Quels sont ses effets et sa performativité sur le spectateur ? Dès le début du reportage This is Climate change: famine, la caméra étant posée au ras du sol, l'angle de vue produit une puissante sensation d'écrasement et place le destinataire à terre sur un sol désertique, face à la vision macabre d'une carcasse d'animal desséché qui semble le regarder à travers des orbites pourtant vides. Puis, très vite, il se retrouve à hauteur d'homme, interpellé directement par les personnes filmées vivant dans ce désert aride, qui semblent vouloir engager un dialogue. Comme le note Émilie Ropert-Dupont, de nombreuses

17 Le reportage This is Climate Change: Famine, créé par Danfung Dennis et Eric Strauss, propose une immersion parmi les réfugiés climatiques du nord de la Somalie, déplacés en raison de la sécheresse de 2017. Il est disponible à : https://www. with.in/watch/this-is-climate-change-famine 
productions immersives se donnent pour objectif de déconstruire une « rhétorique de la déshumanisation » (Ropert-Dupont, 2017, p. 168). Ici, le procédé narratif agit comme un récit initiatique métaphorique où le destinataire prend la place de celui qui souffre de la faim. Le destinataire, d'abord à terre, est comme la carcasse face à lui qui semble refléter sa condition imaginaire d'être humain touché par la famine : écrasé par la souffrance, abandonné, nié dans son humanité et ramené à une animalité et à une mort prochaine. Seuls l'interpellation, figure connue de la subjectivation, et le dialogue avec ses semblables, la considération de l'autre qu'il oblige par la parole et le regard, le réintègrent dans la société des humains. Au fond, c'est ce que ce reportage fait espérer : que le destinataire voie l'autre comme dans un miroir, et qu'il puisse le considérer, celui qui souffre de la faim, dans toute son humanité, en faisant un geste pour lui venir en aide. Le reportage immersif porte avec lui cette idée que la vérité n'est pas qu'un discours mais une expérience reposant sur l'émotion. Cela participe au mode de « véridiction » propre à ce dispositif, qui consiste à énoncer la vérité, à l'asséner, ce qui se fait ici à travers une médialité de la transparence. Le reportage immersif pourrait « réussir », en regard de ses prétentions « épistémiques » et par son mode de véridiction, à convaincre de sa véracité et de son authenticité. Mais, est-ce suffisant pour faire perdre cette étrangeté et cette distance aux personnages de This is Climate change: famine ? Sont-ils, grâce à l'immersion, davantage perçus comme des êtres humains semblables à l'utilisateur? L'émotion est-elle la condition suffisante à l'empathie ? Le destinataire, réduit à la présence fantomatique d'un regard, n'a ni la possibilité d'interagir avec les protagonistes, ni de véritable liberté de mouvement (Toursel \& Useille, 2019). Il lui est impossible de nouer une pleine relation pratique avec l'environnement proposé. L'expérience immersive du reportage n'échouerait-elle pas dans sa prétention à faire « monde » au sens éthique et politique (Wolff, 2014) ?

\section{Une ontologie paradoxale}

La question abordée précédemment fait nettement ressortir l'authenticité et la véracité de l'information comme modes de vérité du reportage immersif. Cette question résulte d'une investigation d'ordre épistémologique tandis que celle qui touche le statut de la réalité à laquelle nous fait accéder le reportage immersif est d'ordre ontologique, ces deux ordres étant liés. L'épistémologie du journalisme, en tant qu'étude des actualités et du journalisme comme une forme spéci- 
fique de savoir sur le monde, pose cette question : « quelle connaissance du monde les consommateurs d'informations obtiennent-ils par le biais du journalisme ? » (Ekström \& Westlund, 2019a, p. 2) ${ }^{18}$. Quant à l'ontologie, elle interroge le positionnement ontologique sous-jacent aux théories et aux pratiques : qu'entend-t-on précisément par « monde »? À quel «monde » accède-t-on à travers le journalisme?

Les discussions philosophiques de ces dernières années, portées par un regain d'intérêt à l'égard de la vérité et du réel, ont renouvelé la question ontologique : « la pensée contemporaine est traversée par un puissant désir réaliste » (Alloa \& During, 2018, p. 14). Cet apport philosophique nourrit des débats en sciences humaines. Pour résumer schématiquement, ils prennent la forme d'une dualité théorique entre constructivisme et (néo)réalisme. D'une part, le constructivisme postule que le réel connaissable est construit, dès lors, il n'est pas possible de s'appuyer sur une « norme du vrai » (Le Moigne, 2012, p. 45), ni même de prétendre accéder à une quelconque vérité. Le (néo)réalisme propose, d'autre part, un renouvellement de la théorie réaliste qui, dans la variété des sens dans lesquels elle s'entend, postule que le réel est donné, indépendamment des conceptions et des théories que nous formulons à son sujet. De la sorte, nous pouvons connaître les choses telles qu'elles sont dès lors que nous faisons correspondre ces conceptions et théories à ce qui est (Gabriel, 2015).

Ce débat s'est étendu jusque chez les théoriciens du journalisme pour penser l'ontologie de l'information journalistique (Fleury-Vilatte \& Walter, 2004). En journalisme, dans son étude théorique comme dans sa pratique, le paradigme constructiviste considère que le réel se caractérise par « son indétermination initiale du point de vue du sens » (Delforce, 2004, p. 115), les faits que traite le journaliste sont inscrits dans un contexte social et discursif, il n'y a pas de fait brut. De son côté, le paradigme d'inspiration (néo)réaliste admet évidemment que l'information journalistique est construite (Gauthier, 2016). Toutefois, la vérité étant un impératif du journalisme, le réalisme épistémologique du journalisme implique conséquemment un réalisme ontologique : il lui faut reconnaître qu'il existe une réalité préalable et que « les faits journalistiques ne sont pas érigés ex nihilo mais toujours à partir de faits bruts et donc non construits »(Gauthier, 2005). En ce sens, des faits bruts composent un donné pré-journalistique à partir duquel sont

18 Notre traduction de « What knowledge of the world do people get as news audiences and through journalism? » (Ekström \& Westlund, 2019a, p. 2). 
construites les nouvelles, c'est-à-dire les faits journalistiques construits. Sans prétendre trancher dans ce débat, utiliser, dans une visée heuristique, la distinction entre fait brut et fait journalistique introduite par le (néo)réalisme permet de poser à nouveaux frais la question du statut de la réalité de l'information journalistique proposée à travers les reportages immersifs.

Les discours des journalistes accompagnant les reportages immersifs prétendent qu'ils donnent accès au destinataire à la réalité brute et sans filtre de l'événement. Si l'immersion est bien réussie et que la médiation tend à s'effacer en tant que telle, le destinataire pourra peut-être se croire transporté « ailleurs et alors » : comme si cette vidéo était le fait brut, comme s'il prenait la place du journaliste plongé dans un événement dont les ressorts sont encore flous, comme si ce reportage n'était pas le résultat d'une construction. L'expression « comme si » nous ramène pourtant aux limites ontologiques qui séparent le fait brut et sa représentation dans le fait journalistique construit. Ce que produisent ces nouvelles médiations reste, comme pour toute production journalistique, une re-présentation, qui tient lieu d'autre chose, voire même qui serait autotélique, n'ayant d'autre fin qu'elle-même : le destinataire jouirait dans certains cas d'un spectacle immersif clos sur lui-même dans un dispositif qui l'isole, et où " le retrait du monde », pour reprendre les termes de Jonathan Crary, est la condition étrange de l'immersion dans l'actualité. Le fait brut devient une trace à partir de laquelle se fabrique le fait journalistique. Celui-ci est en fait très travaillé : les images sont « cousues » lors du montage pour constituer une narration, guidant le regard du destinataire. Ainsi, par la superposition des niveaux expérientiels, tels que le vivent ceux qui sont submergés par l'événement et ceux qui sont immergés dans l'image, le reportage immersif brouille les frontières des évènements scindés entre le fait brut que le spectateur croit vivre (mais le croit-il vraiment ?), et l'information journalistique qu'il vit réellement et qui est doublement construite, par le journaliste d'abord, puis encore re-construite par l'utilisateur luimême, au gré de son expérience singulière. Au cours de son exploration de ces supposés faits bruts, le destinataire est enjoint à donner du sens à ce qu'il vit, à partir d'une expérience médiatique en fait, brouillant les frontières du débat entre constructivisme et réalisme. 


\section{Conclusion}

Dans quelle mesure le reportage immersif propose-t-il une expérience paradoxale du réel et de la vérité ? Le destinataire est invité à entrer dans l'image, à s'y mouvoir, à interagir avec elle pour vivre l'actualité. Ce journalisme s'inscrit dans une longue tradition d'expériences immersives développées dans les arts visuels, mais aussi en sciences sociales. Le reportage immersif qui vise à transporter le spectateur sur le terrain d'enquête, se prête bien à une telle expérimentation, apte à toucher le public en proposant une expérience subjective de l'information. Le lecteur des reportages écrits par Greenwood, Nellie Bly et Stephen Crane est certainement lui aussi transporté dans une situation qui lui est inconnue. De même, quand James Nachtwey photographie la douleur, l'injustice et la violence, il parvient par d'autres moyens à restituer avec force son témoignage pour que son destinataire prenne acte, non plus comme spectateur mais comme citoyen, de «l'entière responsabilité d'être là » comme il le déclare (Nachtwey, 2018, p. 5). Néanmoins, le journalisme immersif semble vouloir aller plus loin en cultivant une médialité de la transparence pour mettre en situation le spectateur et lui faire vivre l'actualité. Ce type de journalisme pourrait être considéré comme le symptôme ou la conséquence de l'ère dite de la "post-vérité », car il saisit la réalité à travers le mode de l'expérience singulière et de l'émotion.

Le reportage immersif est un objet composite où la technologie prend place dans une configuration discursive et culturelle plus vaste qui implique une certaine idée du journalisme dans sa relation à la réalité et à la vérité, dans sa manière de concilier la transparence des médias et la distance de la médiation, formule que le reportage immersif pousse jusqu'au paradoxe.

On croit que celui-ci trouve sa résolution dans la présence active du destinataire dans l'image qui agit comme une instance d'authentification de ce qui a été « rapporté » puisqu'il éprouverait à son tour ce que le journaliste a éprouvé en situation. Mais en lui donnant à vivre de supposés faits bruts, le destinataire est confronté avant tout à une représentation médiatique qui lui fournit le cadre d'une expérience reconstruite de la réalité.

Le reportage immersif aboutit à des conséquences contradictoires et étonnantes à propos de l'information produite et donne lieu à une expérience singulière et personnelle, très éloignée de la réalité de l'autre qu'il était supposé nous faire vivre. Tout son intérêt réside dans 
les tensions, les paradoxes et les dissonances qu'il recèle et où se lisent en filigrane les évolutions mais aussi les enjeux des médiacultures et du journalisme contemporain.

\section{Références}

Aïm, O. (2013). Le transmédia comme remédiation de la théorie du récit. Terminal 112, 43-55. doi : https://doi.org/10.4000/terminal.558

Alloa, E. \& During, E. (Éd.) (2018). Choses en soi : métaphysique du réalisme. Paris : PUF.

Aubenas, F. (2010). Le Quai de Ouistreham. Paris : Éditions de L'Olivier.

Beyaert-Geslin, A. (2009). L'Image préoccupée. Paris : Hermès-Lavoisier.

Boltanski, L. (2007). La Souffrance à distance : morale humanitaire, médias et politique. Paris : Gallimard.

Brabant, S. (2012). Le Reportage à la télévision. De la conception à la diffusion. Paris : CFPJ Éditions.

Cambier, A. (2019). Philosophie de la post-vérité. Paris : Hermann.

Carlson, M. (2016). Metajournalistic Discourse and the Meanings of Journalism: Definitional Control, Boundary Work, and Legitimation. Communication Theory 26(4), 349-368. doi : https://doi.org/10.1111/comt.12088

Cornu, D. (1998). Journalisme et vérité. Autres Temps. Cahiers d'éthique sociale et politique 5, 13-27. doi : https://doi.org/10.3406/chris.1998.204

Crary, J. (2016). Techniques de l'Observateur. Vision et modernité au XIXe siècle. Bellevaux : Éditions Dehors.

De la Peña, N. et al. (2010). Immersive Journalism: Immersive Virtual Reality for the First-person Experience of News. Presence: Teleoperators and Virtual Environments 19(4), 291-301. doi : https://doi.org/10.1162/PRES_a_00005

Delforce, B. (2004). Le constructivisme : une approche pertinente du journalisme. Questions de communication 6, 111-134. doi : https://doi.org/10.4000/questionsdecommunication. 4345

Ekström, M. \& Westlund, O. (2019a). Epistemology and Journalism. Oxford Encyclopedia of Journalism Studies, Oxford University Press. doi : http://dx.doi. org/10.1093/acrefore/9780190228613.013.806

Ekström, M. \& Westlund, O. (2019b). The Dislocation of News Journalism: A Conceptual Framework for the Study of Epistemologies of Digital Journalism. Media and Communication 7(1), 259-270. doi : https://doi.org/10.17645/mac. v7i1.1763.

Ferraris, M. (2018). Post vérité et autres énigmes. Paris : PUF.

Fleury-Vilatte, B. \& Walter, J. (2004). Des usages du constructivisme (2). Questions de communication 6, 101-110. doi : https://doi.org/10.4000/questionsdecommunication. 4334

Gabriel, M. (2015) Pourquoi le monde n'existe pas. Traduit par Sturm G. et Sturm S. Paris : Librairie générale française.

Gaudreault, A. \& Jost, F. (2005). Le Récit cinématographique, 2e éd. Paris : Nathan.

Gaudreault, A. \& Marion, Ph. (2013). La Fin du cinéma? Paris : Colin. 
Gauthier, G. (2004). La vérité, visée obligée du journalisme. Les Cahiers du journalisme 13, 164-179. Disponible à : http://www.cahiersdujournalisme.net/pdf/13/10 Gauthier.pdf

Gauthier, G. (2005). La réalité du journalisme. Communication 23(2). doi : https://doi. org/10.4000/communication. 4120

Gauthier, G. (2016). Un point de vue néoréaliste en épistémologie du journalisme. Sur le journalisme, About journalism, Sobre jornalismo 5(2), 22-28.

Gleizes, D. \& Reynaud, D. (2017). Machines à voir. Pour une histoire du regard instrumenté, $X V I I^{e}-X I X^{e}$ siècles. Lyon: Presses universitaires de Lyon.

Grau, O. (1999). Into the Belly of the Image: Historical Aspects of Virtual Reality. Leonardo 32(5), 365-371. Disponible à : https://www.digitalartarchive.at/fileadmin/ user_upload/Virtualart/PDF/153_Into_the_Belly_of_the_Image-_Historical_Aspects_of_Virtual_Reality-libre.pdf

Jost, F. (1987). L'Eil-caméra : entre film et roman. Lyon : Presses universitaires de Lyon.

Karlin, B. et al. (2018). Does Medium Matter? Exploring the Role of Virtual Reality in Journalism. The Media Impact Project of the USC Annenberg Norman Lear Center. Disponible à : http://www.mediaimpactproject.org/uploads/5/1/2/7/5127770/frontlinevrreport_final.pdf

Kovach, B. \& Rosenstiel, T. (2014). Principes du journalisme. Ce que les journalistes doivent savoir, ce que le public doit exiger. Paris : Gallimard.

Lancelin, A. (2018). La Pensée en otage : S'armer intellectuellement contre les médias dominants. Paris : Les Liens qui libèrent.

Lancien, T. (2011). Le Journal télévisé. De l'événement à sa représentation. Bordeaux : Presses universitaires de Bordeaux.

Le Cam, F. \& Ruellan, D. (2017). Émotions de journalistes. Grenoble : Presses universitaires de Grenoble.

Le Moigne, J. (2012). Les Épistémologies constructivistes, $4^{\mathrm{e}}$ éd. Paris : PUF.

Leroux, P. \& Neveu, E. (2017). En immersion. Pratiques intensives du terrain en journalisme, littérature et sciences sociales. Rennes: Presses universitaires de Rennes.

Marian, D. (2016). The Correspondence Theory of Truth. The Stanford Encyclopedia of Philosophy. Disponible à : https://plato.stanford.edu/archives/fall2016/entries/ truth-correspondence.

Mercier, A. (2018). Fake news et post-vérité : 20 textes pour comprendre la menace. The Conversation France. Disponible à : https://hal.univ-lorraine.fr/hal-01819233/ document.

Mercier, A. \& Pignard-Cheynel, N. (2014). Mutations du journalisme à l'ère du numérique : un état des travaux. Revue française des sciences de l'information et de la communication 5. doi : https://doi.org/10.4000/rfsic.1097

Mersch, D. (2018). Théorie des médias. Une introduction. Dijon : Les Presses du Réel.

Nachtwey, J. (2018). Memoria, photographies de James Nachtwey. Maison Européenne de la Photographie. Paris : Contrasto.

Parikka, J. (2017). Qu'est-ce que l'archéologie des médias? Grenoble : UGA Éditions.

Revault d'Allonnes, M. (2018). La Faiblesse du vrai. Ce que la post-vérité fait de notre monde commun. Paris : Seuil.

Ropert-Dupont, É. (2017). Journalisme et réalité virtuelle : Émotion ou information. Paris : L'Harmattan. 
Toursel A. \& Useille, P. (2019). Immersive Journalism: A "New Frontier" of Information Experience? Brazilian Journalism Research 15(2). doi : https://doi. org/10.25200/BJR.v15n2.2019.1230

Useille, P., Lamy, A. \& Blanchard, G. (2017). Les médias d'information jouent-ils le jeu de l'innovation? Le cas du newsgame. RIHM Revue des Interactions Humaines Médiatisées 18(2), 57-73. Disponible à : http://europia.org/RIHM/V18N2.htm.

Vanoost, M. (2017). Les expériences de Stephen Crane ou l'articulation d'une conception originale et complexe de l'immersion journalistique. Dans P. Leroux \& E. Neveu (2017). En immersion. Pratiques intensives du terrain en journalisme, littérature et sciences sociales (pp. 37-49). Rennes : Presses universitaires de Rennes.

Wallraff, G. (1986). Tête de turc. Le destin des immigrés en situation irrégulière dans la Ruhr. Paris : La Découverte.

Williams, B. (2006). Vérité et véracité : essai de généalogie. Paris : Gallimard.

Wolff, F. (2014). Dire le monde. Paris : PUF.

\section{(c) $(1) \Theta \Theta$} «Attibution - pas d'utilisation Commerciale - Pas de Modification 4.0. International» (CC BY-NC-ND) 